\title{
Mesenchymoma of the lung (so called hamartoma): a review of 154 parenchymal and endobronchial cases
}

\author{
J M M VAN DEN BOSCH, Sj Sc WAGENAAR, B CORRIN, J R J ELBERS, \\ P J KNAEPEN, C J J WESTERMANN
}

From the Department of Pulmonary Disease, Pathology, and Cardiothoracic Surgery, St Antonius Hospital, Nieuwegein, The Netherlands, and the Department of Thoracic Pathology, Cardiothoracic Institute and Brompton Hospital, London

ABSTRACT In a series of 154 patients (116 male and 38 female) with so called pulmonary hamartoma the peak incidence was in the sixth decade, with only three patients less than 20 years of age ${ }_{-}^{\supset}$ Sequential radiographs showed that in 55 patients the tumour first appeared in adult life and that in 53 it progressively increased in size. The age incidence and progressive growth leads to the conclusion that the tumour is a benign neoplasm rather than a hamartoma, consisting of various connective tissues intersected by clefts lined by respiratory epithelium. The epithelial elements are regarded as entrapped non-neoplastic inclusions and the tumour as a purely mesenchymal neo 0 plasm: the name mesenchymoma therefore seems the most appropriate. There were two recurrences after simple enucleation, 10 and 12 years later. A total of 142 tumours were parenchymal, and onlye 12 were endobronchial. All lobes were affected but there was a slight preponderance in the left upper lobe. Four patients had two (synchronous) mesenchymomas. There was an associated bronchiaf carcinoma in 11 patients, synchronous in six and metachronous in five.

A hamartoma is a non-neoplastic tumour like malformation, ${ }^{1}$ but there is evidence that the so called hamartoma of the lung is a benign neoplasm. ${ }^{2} \mathrm{We}$ have reviewed the clinical and pathological features of 154 patients with this entity and present data supporting the argument that it is.a true neoplasm. We prefer to classify these lesions as benign mesenchymal tumours of the lung or mesenchymomas.

\section{Clinicopathological data}

\section{PATIENTS}

Mesenchymoma was diagnosed in 158 patients from 1956 to 1984 , but the histological slides of only 154 were available for review. The patients were drawn from a predominantly Dutch population of about 500000 , representing an annual incidence of about 1:100000. They ranged in age from 14 to 74 years (mean 51 years), with a peak incidence in the sixth decade. The tumour was detected before the third

Address for reprint requests: Dr J M M van den Bosch, Department of Pulmonary Disease, Sint Antonius Hospital, PO Box 2500, 3420 EM Nieuwegein, The Netherlands.

Accepted 23 February 1987 decade in only three patients, aged 14,15 , and 172 years. The male to female ratio was $3: 1$. Smoking histories had seldom been obtained. In $142(92 \%)$. patients the tumour was parenchymal, and in only 125 $(8 \%)$ was it visible with the rigid bronchoscope. Alf lobes were affected, with a slight preponderance in the left upper lobe. Four patients were found to have two synchronous tumours.

One patient with a parenchymal lesion presented with a small haemoptysis. The remainder were symptomless, the tumours being incidental findings in? routine chest radiographs. Ten of the 12 patients with endobronchial mesenchymomas, on the other hand had pulmonary symptoms in the three monthso leading up to the diagnosis, including cough ( 9 cases) $N$ phlegm (4), haemoptysis (3), dyspnoea on exertionn (6), and fever (3).

\section{RADIOGRAPHY}

Preoperative chest radiographs were available fromes 131 patients. Parenchymal mesenchymomas formed a peripheral opacity, which was round in $80 \%$ of cases: and lobulated in $20 \%$. The opacity was homo- $\vec{D}$ geneous in all but 13 cases that showed partia? calcification; it was eccentric in 10 and central in three. The chest radiographs of nine patients with 
endobronchial mesenchymoma showed either segmental atelectasis (5), no abnormality (1), consolidation (1), hyperinflation of the lobe (1), or a patchily calcified tumour (1).

Previous chest radiographs were available from 57 patients. In 30 the tumour was not visible one to 18 years before detection. In 25 cases radiographs taken one to 13 years before diagnosis showed a tumour that had since increased in size. In only two patients did previous chest radiographs, one and three years before detection, fail to show enlargement of the tumour.

\section{ADDITIONAL TUMOURS}

Five patients had an additional opacity that later proved to be a simultaneous bronchial carcinoma, and a further patient developed a bronchial carcinoma within six months. The carcinoma was squamous in three cases, adenocarcinoma in two cases, and adenosquamous in one. All but one affected the same lobe as the mesenchymoma. A further patient had a simultaneous retroperitoneal phaeochromocytoma, but was not thought to have an incomplete Carney's triad (bronchial chondroma, multiple gastric leiomyosarcomas and extra-adrenal paraganglioma) because of her age (66 years), the parenchymal location of the pulmonary tumour, and its microscopic structure.

\section{FEATURES OF THE MESENCHYMOMAS}

In three patients with endobronchial mesenchymoma the preoperative pathological diagnoses were chondroma, lipoma, and granulation tissue. The correct diagnosis was made in two instances. Bronchoscopic examination did not provide a diagnosis in the remaining seven patients with endobronchial growths or in any of the patients with parenchymal mesenchymoma.

Histology of endobronchial tumours The endobronchial tumours varied from 8 to $70 \mathrm{~mm}$ in diameter (mean $21 \mathrm{~mm}$ ), with most in the $10-30 \mathrm{~mm}$ range. The connective tissue component was predominantly chondroid in $50 \%$, fatty in $33 \%$, fibroblastic in $8 \%$, and osseous in $8 \%$. The chondroid tissue bore no anatomical relationship to the airway cartilage but the osseous component always occurred in the chondroid element and appeared to be metaplastic. Young, active fibroblasts, often of irregular configuration, were usually located close to the chondroid elements. Seromucous glands were scattered between the fatty or fibroblastic components and the surface of the tumour was covered by respiratory epithelium. In nine cases the surface was smooth, but in two a papillary structure with the formation of epithelial inclusions was seen. The chondroid tissue was usually nodular, giving the tumour a lobulated character, probably reflecting multicentric maturation.

Histology of parenchymal tumours The parenchymal tumours varied in size from 4 to $90 \mathrm{~mm}$ in diameter (mean $19 \mathrm{~mm}$ ). In $80 \%$ the predominant differentiation was chondroid, in $12 \%$ fibroblastic, in $5 \%$ fatty, and in $3 \%$ osseous. The boundary between tumour and lung was often indistinct or papillary, as a result of outgrowths of fibroblasts into alveolar walls. This was particularly evident in the tumours with multicentric maturation; such tumours also showed epithelial inclusions of type II pneumocytes, or ciliated, non-ciliated, or mucus-producing bronchiolar cells. Other parenchymal tumours had a more solid appearance, with a sharp border and few epithelial inclusions; these had a predominantly chondroid structure. At the periphery of the tumour lymphocytes, plasma cells, and occasionally mast cells were often present. Collections of macrophages were usually present in adjacent alveolar spaces, and in one case the appearances around the tumour were typical of a plasma cell granuloma. Three peripheral tumours were associated with non-caseating granulomas in which no microorganisms could be detected; there was no evidence of sarcoidosis, either at the time of thoracotomy or at follow up.

Except for the proportions of the various components, no difference was found between the endobronchial and parenchymal tumours. Moreover, 11 tumours with radiologically documented growth in the year preceding thoracotomy were no different histologically, either in composition or in peripheral extension. In three of the four patients with two tumours, the relative proportions of their components were similar, but in the fourth patient one tumour was predominantly cartilagenous and the other fibrous. Malignant change was not seen. Two patients suffered a recurrence but no difference was found between their first and second tumours, chondroid tissue being the predominant element in both.

\section{TREATMENT AND FOLLOW UP}

Treatment of 129 patients with parenchymal mesenchymoma consisted of thoracotomy with enucleation or wedge excision after frozen section had shown that the lesion was benign. Larger resectionssegmentectomies, lobectomies, or bilobectomieswere performed in 17 patients, because of bronchial carcinoma (5), suspected carcinoma (2), tumour size (1), or central localisation (4); in five the reason for the resection was not documented. Of the 12 patients with endobronchial mesenchymoma, five had a lobectomy, four a segmental resection, and one a lobectomy with reconstructive surgery of the bronchus; one was treated by bronchoscopic removal, and one by thoracotomy and bronchotomy. 
Follow up of 138 patients with parenchymal mesenchymoma was possible (1-25 years, mean 5). In two patients the tumour recurred in the same pulmonary segment, 10 and 12 years later respectively. Five patients developed a bronchial carcinoma between one and seven years after diagnosis of mesenchymoma, four of which were squamous and one adenocarcinoma. In contrast to the synchronous carcinomas these metachronous growths affected different lobes from the mesenchymoma.

\section{Discussion}

Whereas a hamartoma ceases to grow when the organ in which it is situated stops growing, a neoplasm shows inexorable growth and compresses the surrounding tissue, in the process generally acquiring a capsule, a feature generally lacking in a hamartoma. Mesenchymomas are most often first diagnosed in the fifth or sixth decade of life, ${ }^{2}$ and seldom in childhood, ${ }^{3}$ a strong argument against their being nonneoplastic malformations. In our series only three were detected in adolescence and none in childhood. Our observation that previous radiographs had frequently been normal indicates that mesenchymoma often develops in middle age. Furthermore, when the tumour had been apparent in previous radiographs it was generally evident that it had increased in size in the interim. In only two patients was there evidence of no continued growth, and in these the follow up period was comparatively short. These features suggest that the lesions are neoplastic rather than hamartomatous.

The pathological evidence is less strong and indeed could well be considered to favour a hamartomatous rather than neoplastic origin. Unlike most expansile tumours, these lesions lack a capsule and show no appreciable compression of the surrounding lung, although in our experience benign neoplasms in pulmonary tissue often lack these features. Furthermore, the mixture of several connective tissue elements with epithelial clefts could be taken to represent a bizarre bronchial maldevelopment, and has been quoted in support of the hamartomatous nature of the lesion. ${ }^{4}$ Entrapment of various pulmonary components, however, is frequently seen in lung tumours, ${ }^{5}$ and in our opinion the epithelial clefts of the so called hamartoma are formed in the same way. We do not favour the frequently used term adenochondroma, but prefer one that reflects a purely mesenchymal histogenesis. Since these lesions consist of various connective tissues we favour the term benign mesenchymal neoplasm or mesenchymoma.

The relationship of mesenchymoma to chondroma, fibroma, and lipoma of the lung has been considered previously and the differences noted. ${ }^{4}$ Chondromas? and lipomas are generally endobronchial, containe only one type of tissue, and lack epithelial inclusions $\frac{\overline{\bar{n}}}{\overline{7}}$ whereas mesenchymomas are more often parenchymal than endobronchial, consist of multiple types of connective tissue, and contain epitheliats inclusions.

Mesenchymomas occur both endobronchially andin the pulmonary parenchyma, the former making upw some $10-20 \%{ }^{26}$ In large series mesenchymomas constitute $7-14 \%$ of all coin lesions. ${ }^{78}$ They are distributed fairly uniformly throughout the lungs $\hat{N}$ Multiple mesenchymomas are rare,, 1011 and thosepatients who had two tumours in our series represent an unusually high proportion. Very rarely both\& endobronchial and parenchymal mesenchymomas are? found in the same patient. ${ }^{9}$ The high prevalence of mesenchymoma in males $^{12}$ possibly reflects the preferential participation of men in mass radiographico्ठ surveys, a common way in which the asymptomatic parenchymal mesenchymoma is detected.

The typical radiological abnormality is a round $\overrightarrow{0}$ homogeneous opacity in the periphery of the lung: $v$ Only occasionally does it appear lobulated, despite the frequency of this pathological finding Calcification is evident radiologically in $10 \%$, particularly at the periphery. Radiological abnormalities in patients with endobronchial tumours are mainly the result of bronchial obstruction.

The surgical treatment was usually enucleation or 3 wedge resection. Extensive resection was performed in? only $12 \%$ of the parenchymal group, whereas in the endobronchial group it was undertaken in $83 \%$ because of the central localisation. Two of ouro patients had a second resection owing to recurrence in the same pulmonary segment, probably due to? incomplete removal. The histological growth pattern suggests that a wedge excision with a narrow margino of normal lung, rather than simple enucleation, may be necessary to prevent recurrence. Multicentric growth could explain apparent recurrences, ${ }^{13}$ but is unlikely because multiple growths are decidedly rare․ㅡㄹ.

Eleven of our patients $(7 \%)$ also had bronchiars carcinoma, which was synchronous in six and? metachronous in five. This association has been observed by others and the risk of lung cancer hasw been estimated to be 6.3 times higher in patients with mesenchymoma than in the general population. ${ }^{14}$ Theo reason for this is not clear, although the developmento of cancer could well lead to the discovery of $\mathrm{an}^{\text {? }}$ ? asymptomatic mesenchymoma that would otherwise not have been detected. The assessment of patients? with mesenchymoma and carcinoma may be difficult, $\stackrel{\mathbb{Q}}{\stackrel{9}{\oplus}}$ because the mesenchymoma can easily be mistaken for a metastasis. 


\section{References}

1 Albrechts E. Ueber Hamartome. Verh Deutsch Ges Pathol 1904;7:153-7.

2 Bateson EM. So called hamartoma of the lung-a true neoplasm of fibrous connective tissue of the bronchi. Cancer 1973;31:1458-67.

3 Hartman GE, Shochat SJ. Primary pulmonary neoplasms of childhood: a review. Ann Thorac Surg 1983;36:108-19.

4 Liebow AA. Tumors of the lower respiratory tract. In: Atlas of Tumor Pathology. Section V, fasc 17. Washington DC: Armed Forces Institute of Pathology, 1952:119.

5 Alvarez-Fernandez E. Alveolar trapping in pulmonary carcinomas. Diagn Histopathol 1982;5:59-64.

6 Thomashefski JF. Benign endobronchial mesenchymal tumors. Their relationship to parenchymal pulmonary hamartomas. Am J Surg Pathol 1982;6:531-40.

7 Steele JD. The solitary pulmonary nodule. $J$ Thorac Cardiovasc Surg 1963;46:21-39.

8 Ray JF, Lawton BR, Magnin GE, et al. The coin lesion story: update 1976, twenty years' experience with early thoracotomy for 179 suspected malignant coin lesions. Chest 1976;79:332-6.

9 Minasian H. Uncommon pulmonary hamartomas. Thorax 1977;32:360-4.

10 Bateson EM. Cartilage-containing tumours of the lung. Relationship between the purely cartilaginous type (chondroma) and the mixed type (so called hamartoma): an unusual case of multiple tumours. Thorax 1967;22:256-9.

11 Muller M, Rijncki PV, Saegesser F, Gardiol D. Hamartomes pulmonaires multiples bilateraux. Schweiz Med Wochenschr 1974;104:511-4.

12 Bateson EM. Relationship between intrapulmonary and endobronchial cartilage-containing tumours (so called hamartomas). Thorax 1965;20:447-61.

13 Anderson MN. Multicentric hamartomas of the lungreport of a case with two additional lesions nine years after primary resection. Ann Thorac Surg 1968;6:469-72.

14 Karasik A, Modan M, Jacob CO, Lieberman Y. Increased risk of lung cancer in patients with chondromatous hamartoma. $J$ Thorac Cardiovasc Surg 1980;80:217-20. 